\title{
Lensing Constraints on Dark Matter Substructure in Galaxies $\left(25^{\prime}+5^{\prime}\right)$
}

\author{
Charles R. Keeton* \\ Department of Physics \& Astronomy, Rutgers University, 136 Frelinghuysen Rd., Piscataway, NJ \\ 08854 USA \\ E-mail: keetonephysics.rutgers.edu
}

\begin{abstract}
The Cold Dark Matter paradigm predicts that galaxy dark matter halos contain a myriad of bound subhalos left over from the hierarchical galaxy formation process. The amount of substructure on different scales is sensitive to the nature of dark matter. Strong gravitational lensing provides the only probe of dark matter substructure in galaxies outside the Local Group. The positions, brightnesses, and time delays of the images in multiply-imaged quasars are sensitive to dark matter subhalos over a wide range in mass. The theory of substructure lensing is rich and tractable, providing a firm foundation for observational studies. Existing data reveal the average amount of substructure, which is consistent with CDM predictions. Future large samples will allow us to measure the evolution of substructure with cosmic time, providing unique access to the distribution of dark matter on small scales, and key astrophysical evidence about the nature of dark matter.
\end{abstract}

Identification of dark matter 2008

August 18-22, 2008

Stockholm, Sweden

\footnotetext{
${ }^{*}$ Speaker.
} 
The Cold Dark Matter paradigm is justly acclaimed for its success explaining cosmological observations relating to the global geometry and expansion history of the universe, and the distribution of matter on large scales and within massive systems like clusters of galaxies. However, there is tension between CDM predictions and observations that probe the distribution of matter inside galaxies. CDM predicts that galaxy dark matter halos should be dense and centrally concentrated, whereas many observations seem more consistent with halos that have low central densities. Also, CDM predicts that each galaxy's halo should contain the intact remnants of thousands of its progenitors (see the contributions by Frenk et al. and Kuhlen et al., and references therein). In the Local Group, the predicted number of dark matter subhalos significantly exceeds the observed number of dwarf galaxy satellites, and the discrepancy is larger at smaller subhalo masses.

The discord shows that we still have a lot to learn about galaxy formation in a dark matter universe. One thing we do not fully understand is the interplay between various processes that determine the amount of (visible) substructure in galaxy halos: the accretion of new subhalos from the environment, versus the destruction of old subhalos by tidal forces [1]-3]; and the extent to which galaxy formation in low-mass systems may be inefficient, or even squelched by photoionization [4, 5]. Another aspect of the problem relates to the nature of dark matter itself. Of the various dark matter candidates that are compatible with the large-scale structure of the universe, some yield different predictions about the distribution of matter on small scales. In particular, models that lead to a suppression of small-scale power reduce the amount of substructure in galaxies and create a signature in the substructure mass function [1, 6]. Studying dark matter substructure in galaxies therefore provides unique constraints on the astrophysics of galaxy formation on small scales, and circumstantial but nonetheless important evidence about the nature of dark matter.

Strong gravitational lensing provides the only "direct" gravitational probe of dark matter substructure in galaxies beyond the Local Group. By Fermat's principle, when light from a background source is bent by a foreground lens galaxy with projected gravitational potential $\phi$ (given by the 2-d Poisson equation $\nabla^{2} \phi=2 \Sigma / \Sigma_{\text {crit }}$ where $\Sigma_{\text {crit }}$ is the critical surface density for lensing), the images we see form at stationary points of the "time delay surface,"

$$
\tau(\vec{x})=\frac{1+z_{l}}{c} \frac{D_{o l} D_{o s}}{D_{l s}}\left[\frac{1}{2}|\vec{x}-\vec{u}|^{2}-\phi(\vec{x})\right]
$$

where $\vec{u}$ is the angular position of the light source with respect to the center of the lens, the D's are angular diameter distances between the observer, lens, and source, and $\tau$ gives the excess travel time compared with a hypothetical light ray that travels directly from the source to the observer with no deflection. Since the image positions are given by $\nabla \tau(\vec{x})=0$, they depend on first derivatives of the lens potential. The brightnesses of the images are determined by the curvature of the time delay surface, so they depend on second derivatives of $\phi$. The differential time delays between the images depend on $\phi$ itself. (See the review of strong lensing by Kochanek [7] for more details.)

Any small-scale structure in the lens galaxy makes its presence felt through its effect on $\phi$. For example, Fig. 11 shows that a small mass clump near a lensed image can produce extra light bending on a scale set by the Einstein radius of the clump, $R_{E} \propto m^{1 / 2}$. If the clumps are dark matter subhalos the perturbations have angular scales of milli-arcseconds and we call the phenomenon "millilensing" [8-10]. If the clumps are stars the scales are micro-arcseconds and we call it "microlensing" [7, 11]. In practice the spatial perturbations are usually too small to resolve, but the 

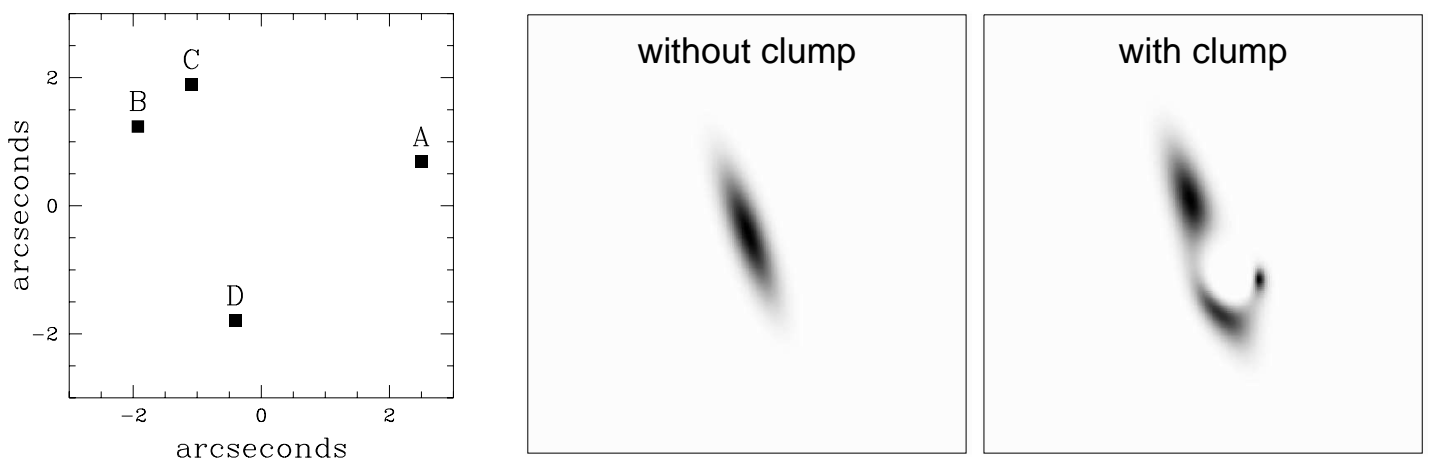

Figure 1: A sample 4-image lens configuration. The middle and right panels show close-ups $\left(0.04^{\prime \prime}\right.$ on a side) of image A with or without a $10^{6} M_{\odot}$ clump nearby. We cannot usually resolve the distortion, but we can detect the change in flux.

concomitant changes in the image brightnesses are very apparent. (Conceptually, $\nabla^{2} \phi$ can have larger fluctuations than $\nabla \phi$ due to small-scale structure.)

We do in fact see many lens systems in which the flux ratios between the images do not follow expected patterns. Keeton et al. [12, 13] introduced a model-independent method to analyze the flux ratios in 4-image lenses with "cusp" and "fold" configurations, which is based on mathematical relations among the lensing magnification factors that are universal for smooth mass distributions. They found that fully half of all cusp and fold lenses violate the theoretical magnification relations, which means the systems are inconsistent with lensing by a smooth mass distribution.

To take the next step and identify what causes the flux ratio anomalies, we need to consider the wavelength of the observations. At optical and X-ray wavelengths, quasar emission regions are small enough that flux ratios can be perturbed by stars in the lens galaxy. As a result, microlensing contributes a significant "background" signal in optical and X-ray data. Many observed optical and X-ray flux ratio anomalies are in fact consistent with pure microlensing [11, 14, 15], although the possibility that there is millilensing as well has not been ruled out. At radio wavelengths, by contrast, the quasar source is thought to be large enough to smooth over the effects of stars and suppress microlensing. Radio flux ratios have thus far been the tool of choice for studying millilensing. Dalal \& Kochanek [10] found that the flux ratios in seven 4-image radio lenses imply that the fraction of mass in substructure is $f_{\text {sub }} \sim 0.02$ (with $0.006<f_{\text {sub }}<0.07$ at $90 \%$ confidence). This amount of substructure is broadly consistent with CDM predictions.

It is important to consider whether millilensing by dark matter substructure is the only viable explanation of (radio) flux ratio anomalies. Evans \& Witt [16] suggested that allowing small departures from elliptical symmetry, modeled with multipole terms, might eliminate the need for lumpy substructure. That turns out not to be the case, however. In some lenses the flux ratio anomalies are so strong that the required multipole terms are completely unrealistic [17]. In other lenses there are additional data that constrain the multipole amplitudes to be too small to explain the flux ratio anomalies [18-20].

As noted above, the mass function $d N / d m$ of substructure may contain information about the nature of dark matter. Unfortunately, it is difficult to constrain the mass function with individual flux ratio measurements because of a degeneracy: a given flux ratio anomaly can be produced by 

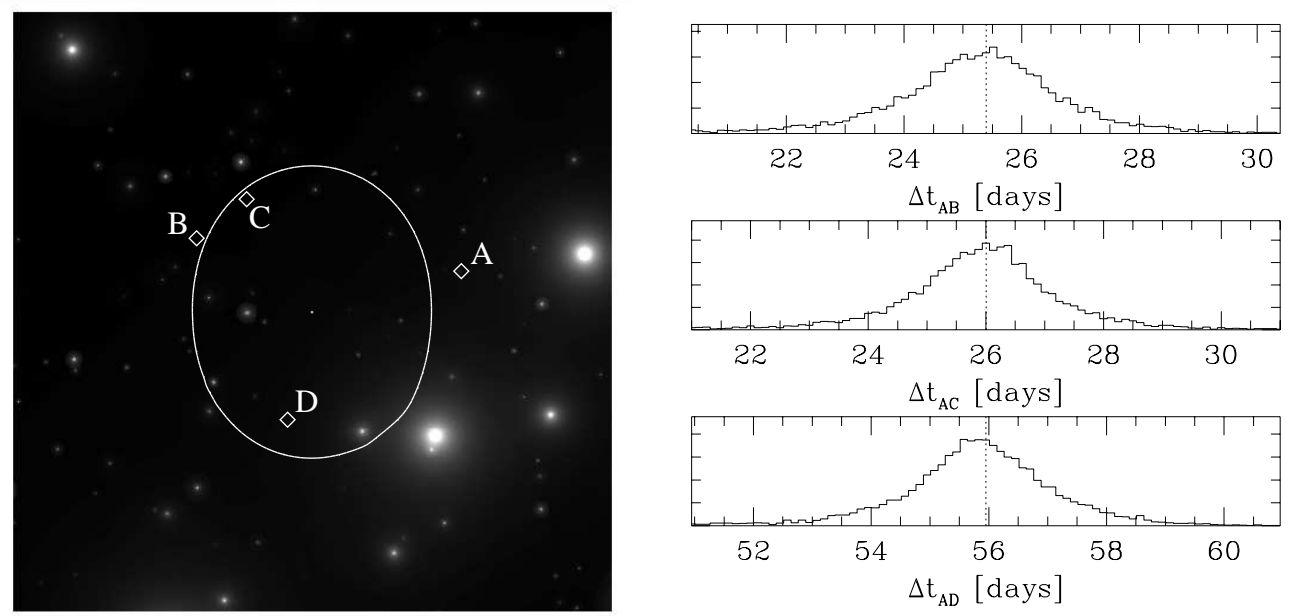

Figure 2: (Left) Sample mass map of substructure from the models by Zentner \& Bullock [1, 2]. The curve shows the lensing critical curve for the full lens model (including the main galaxy, which is omitted from the mass map for clarity). The points show sample lensed images. (Right) Histograms of the time delays between the images, for $10^{4}$ Monte Carlo simulations of the substructure. (The source position is fixed, so the image configuration remains nearly fixed with only small perturbations to the image positions.) The dotted lines show what the time delays would be if all the mass were smoothly distributed.

a low-mass clump very close to an image, or a more massive clump farther away, or anything in between (see eq. 3 below). This degeneracy is broken at low masses because the source only "feels" clumps with Einstein radii $R_{E}(m) \gtrsim R_{\text {src }}$. Heuristically, we can think of flux ratio anomalies as measuring the total amount of substructure above some threshold set by the size of the source,

$$
M_{\mathrm{sub}}\left(R_{\mathrm{src}}\right) \sim \int W\left(R_{E}(m) \mid R_{\mathrm{src}}\right) m \frac{d N}{d m} d m
$$

where $W$ is a weight function that is unity for $R_{E}(m) \gg R_{\text {src }}$ and zero for $R_{E}(m) \ll R_{\text {src }}$ (but may be complicated in between [21]). One idea for constraining the mass function is to measure flux ratios at different wavelengths that correspond to different source sizes [22, 23]. This approach looks promising, although the conclusions will depend on understanding both how quasar size varies with wavelength and how the weight function behaves in the region $R_{E}(m) \sim R_{\text {src }}$.

Keeton \& Moustakas [24] recently suggested that a complementary way to probe the subhalo mass function is to consider a different lens observable, namely the time delays between the images. To illustrate how substructure affects time delays, Fig. 2 shows a sample substructure mass map and mock lens image configuration, plus histograms of the time delays between the images for $10^{4}$ Monte Carlo simulations of the substructure. Even though substructure accounts for just $0.15 \%$ of the mass projected within the galaxy's Einstein radius, it has a substantial effect: the time delays clearly differ from what they would have been if all the mass were smoothly distributed, with a typical scatter of more than a day.

A few remarkable lenses have time delays that already suggest the presence of substructure [25, 26]. More often, we expect that detecting substructure effects will require time delays with uncertainties at the level of \pm 0.3 days or better. This is a factor of $\gtrsim 2$ more precise than most time delays known today (see [27] for a compilation), but feasible in the near term for a modest 

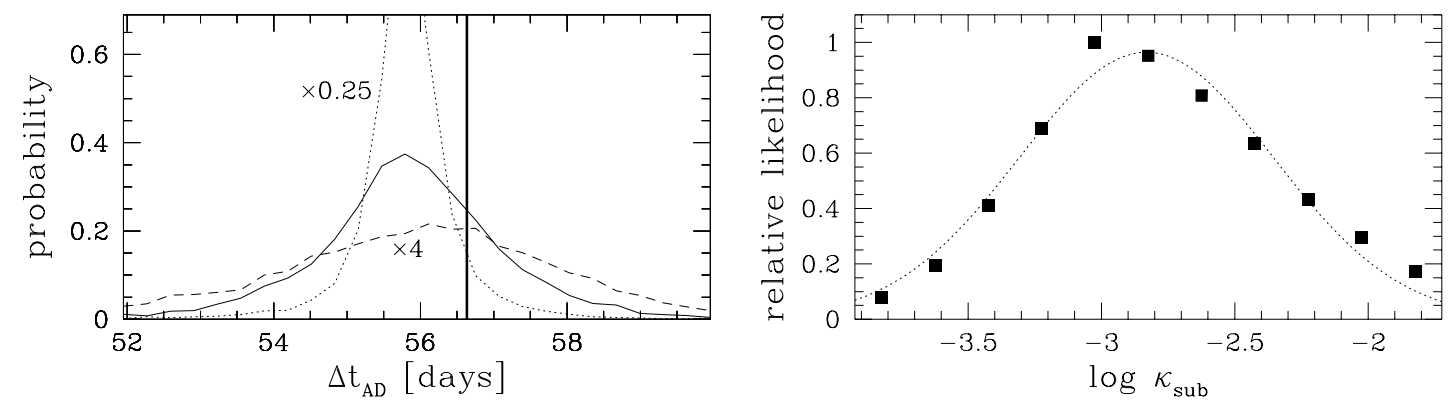

Figure 3: (Left) Time delay distributions for models with different amounts of substructure, for the mock lens shown in Fig. 2. The solid curve shows the reference model, with $\log \kappa_{\text {sub }}=-2.82$, while the dashed and dotted curves have a factor of 4 more or less substructure, as indicated. The vertical line shows a sample value drawn from the reference model. The probability at the "observed" time delay gives the likelihood of each model. Models with too little or too much substructure are disfavored in this likelihood sense. (Right) Repeating the analysis for more models, we can trace the likelihood as a function of $\kappa_{\text {sub }}$ (points). The likelihood function is approximately Gaussian in $\log \kappa_{\text {sub }}$ (dotted curve), with mean $\log \kappa_{\text {sub }}=-2.83$ and dispersion 0.47 dex.

sample of lenses; and the sample will increase substantially over the next 5-10 years [28, 29]. We can then imagine using time delays to constrain the amount of substructure. Heuristically, we will get a lower bound because when there is too little substructure the observed time delay anomalies will be statistically unlikely, and an upper bound because when there is too much substructure the anomalies should (statistically speaking) be even stronger than observed. We can formalize this idea with a likelihood analysis, as illustrated in Fig. 3. For a mock quad lens with time delay uncertainties of \pm 0.3 days, the maximum likelihood method yields $\log \kappa_{\text {sub }}=-2.8 \pm 0.5$, where $\kappa_{\text {sub }}$ is the mean surface mass density in substructure at the positions of the images, in units of the critical density for lensing. This is in excellent agreement with the amount of substructure used to generate the mock data. The uncertainty of $0.5 \mathrm{dex}$ (a factor of 3 ) is admittedly idealized, but there are no obvious effects that would make real lenses substantially worse.

Even more promising is the prospect of combining time delays with flux ratios. The two observables are nicely complementary as probes of substructure, which we can understand as follows. Magnification perturbations are mediated principally through the shear perturbations [8]

$$
\Delta \gamma_{c}=-\sum_{i} \frac{\cos 2 \theta_{i}}{\hat{r}_{i}^{2}}, \quad \Delta \gamma_{s}=-\sum_{i} \frac{\sin 2 \theta_{i}}{\hat{r}_{i}^{2}}
$$

where $\left(r_{i}, \theta_{i}\right)$ are the polar coordinates of a clump relative to the lensed image, $\hat{r}_{i}=r_{i} / R_{E, i}$ is the radius scaled by the clump's Einstein radius, and the sums are over the population of clumps. Time delay perturbations are created principally by the perturbation to the lens potential [24]

$$
\Delta \phi=\sum_{i} R_{E, i}^{2} \ln \hat{r}_{i}
$$

The first conceptual point is that the shear perturbations do not depend explicitly on the Einstein radius, and hence on the clump mass; this is why (individual) flux ratios do not probe the subhalo mass function. By contrast, the potential perturbation has a factor of $R_{E, i}^{2} \propto m_{i}$, which means that 
time delay perturbations are explicitly sensitive to the clump mass. Going into more detail, Keeton \& Moustakas [24] show that the RMS time delay perturbation scales as

$$
\sigma_{t} \propto\left(f_{\mathrm{sub}} \frac{\left\langle m^{2}\right\rangle}{\langle m\rangle}\right)^{1 / 2},
$$

where the averages are taken over the subhalo mass function. An important corollary is that time delay perturbations due to stars are vanishingly small, so there is no microlensing contribution to time delay anomalies.

A second conceptual point is that shear perturbations scale as $\hat{r}^{-2}$, so they are quasi-local, dominated the few nearest subhalos, and potentially of order unity. By contrast, potential perturbations scale as $\ln \hat{r}$, so they are much more long-range and sensitive to collective effects from the full clump population. The factor of $R_{E, i}^{2} \propto m_{i}$ makes the potential perturbation from any given clump fairly small; but the combination of effects from many clumps brings the net time delay perturbation up to a level that is measurable.

To summarize, the evidence is strong that lens galaxies contain small-scale structure, which is consistent with being CDM substructure. To further test CDM predictions and begin to probe the nature of dark matter, we are beginning to investigate the substructure mass function using multiwavelength flux ratios as well as time delays. The different observables contain complementary information about the subhalo population, so they should be very valuable in combination. Useful datasets are available now, and ongoing and planned astronomical surveys should provide large samples that will revolutionize this unique study of dark matter substructure in distant galaxies.

\section{Acknowledgments}

I would like to thank Art Congdon, Greg Dobler, Ross Fadely, and Leonidas Moustakas for their collaboration on various projects related to substructure lensing. I would also like to thank James Bullock and Andrew Zentner for providing some of their substructure models. This work is supported by by NSF grant AST-0747311.

\section{References}

[1] Zentner, A. R., \& Bullock, J. S., Halo Substructure and the Power Spectrum, ApJ 598, 49 (2003).

[2] Zentner, A. R., et al., The Physics of Galaxy Clustering. I. A Model for Subhalo Populations, ApJ 624, 505 (2005).

[3] Kravtsov, A. V., Gnedin, O. Y., \& Klypin, A. A., The Tumultuous Lives of Galactic Dwarfs and the Missing Satellites Problem, ApJ 609, 482 (2004).

[4] Bullock, J. S., Kravtsov, A. V., \& Weinberg, D. H., Reionization and the Abundance of Galactic Satellites, ApJ 539, 517 (2000).

[5] Somerville, R. S., Can Photoionization Squelching Resolve the Substructure Crisis? ApJL 572, L23 (2002).

[6] Colín, P., Avila-Reese, V., \& Valenzuela, O., Substructure and Halo Density Profiles in a Warm Dark Matter Cosmology, ApJ 542, 622 (2000). 
[7] Schneider, P., Kochanek, C. S., \& Wambsganss, J., Gravitational Lensing: Strong, Weak \& Micro, Proceedings of the 33rd Saas-Fee Advanced Course (2006).

[8] Mao, S., \& Schneider, P., Evidence for Substructure in Lens Galaxies? MNRAS 295, 587 (1998).

[9] Metcalf, R. B., \& Madau, P., Compound Gravitational Lenses as a Probe of Dark Matter Substructure within Galaxy Halos, ApJ 563, 9 (2001).

[10] Dalal, N., \& Kochanek, C. S., Direct Detection of Cold Dark Matter Substructure, ApJ 572, 25 (2002).

[11] Kochanek, C. S., et al., Turning AGN Microlensing From a Curiosity Into a Tool, in proceedings of Statistical Challenges in Modern Astronomy IV, ed. G. J. Babu \& E. D. Feigelson (2007).

[12] Keeton, C. R., Gaudi, B. S., \& Petters, A. O., Identifying Lenses With Small-Scale Structure. I. Cusp Lenses, ApJ 598, 138 (2003).

[13] Keeton, C. R., Gaudi, B. S., \& Petters, A. O., Identifying Lenses With Small-Scale Structure. II. Fold Lenses, ApJ 635, 35 (2005).

[14] Blackburne, J. A., Pooley, D., \& Rappaport, S., X-Ray and Optical Flux Anomalies in the Quadruply Lensed QSO 1RXS J1131-1231, ApJ 640, 569 (2006).

[15] Pooley, D., et al., X-Ray and Optical Flux Ratio Anomalies in Quadruply Lensed Quasars. I. Zooming in on Quasar Emission Regions, ApJ 661, 19 (2007).

[16] Evans, N. W., \& Witt, H. J., Fitting Gravitational Lenses: Truth or Delusion, MNRAS 345, 1351 (2003).

[17] Congdon, A. B., \& Keeton, C. R., Multipole Models of Four-Image Gravitational Lenses With Anomalous Flux Ratios, MNRAS 364, 1459 (2005).

[18] Kochanek, C. S., \& Dalal, N., Tests of Substructure in Gravitational Lenses, ApJ 610, 69 (2004).

[19] Yoo, J., et al., The Lens Galaxy in PG 1115+080 is an Ellipse, ApJ 626, 51 (2005).

[20] Yoo, J., et al., Halo Structures of Gravitational Lens Galaxies, ApJ 642, 22 (2006).

[21] Dobler, G., \& Keeton, C. R., Finite Source Effects in Strong Lensing: Implications for the Substructure Mass Scale, MNRAS 365, 1243 (2006).

[22] Chiba, M., et al., Subaru Mid-Infrared Imaging of the Quadruple Lenses PG $1115+080$ and B1422+231: Limits on Substructure Lensing, ApJ 627, 53 (2005).

[23] Poindexter, S., et al., Mid-IR Imaging and a Revised Time Delay for the Gravitational Lens System Quasar HE 1104-1805, ApJ 660, 146 (2007).

[24] Keeton, C. R., \& Moustakas, L. A., A New Channel for Detecting Dark Matter Substructure in Galaxies: Gravitational Lens Time Delays, arXiv:0805.0309.

[25] Morgan, N. D., et al., Time-Delay Measurement for the Quadruple Lens RX J1131-1231, arXiv:astro-ph/0605321.

[26] Congdon, A. B., Keeton, C. R., \& Nordgren, C. E., Using Differential Time Delays to Identify Gravitational Lenses with Small-Scale Structure, ApJ to be submitted (2008).

[27] Oguri, M., Gravitational Lens Time Delays: A Statistical Assessment of Lens Model Dependences and Implications for the Global Hubble Constant, ApJ 660, 1 (2007).

[28] Fassnacht, C. D., et al., Strong Lensing Studies with the LSST, BAAS 36, 1531 (2004).

[29] Marshall, P., Blandford, R., \& Sako, M., The SNAP strong lens survey, NewAR 49, 387 (2005). 